\title{
Semantic Query Transformations for Increased Parallelization in Distributed Knowledge Graph Query Processing
}

\author{
Hyeongsik Kim* \\ Hyeongsik.Kim@us.bosch.com \\ Robert Bosch LLC \\ Sunnyvale, California
}

\author{
Abhisha Bhattacharyya \\ abhatt22@ncsu.edu \\ North Carolina State University \\ Raleigh, North Carolina
}

\author{
Kemafor Anyanwu \\ kogan@ncsu.edu \\ North Carolina State University \\ Raleigh, North Carolina
}

\begin{abstract}
Ontologies have become an increasingly popular semantic layer for integrating multiple heterogeneous datasets. However, significant challenges remain with supporting efficient and scalable processing of queries with data linked with ontologies (ontological queries). Ontological query processing queries requires explicitly defined query patterns be expanded to capture implicit ones, based on available ontology inference axioms. However, in practice such as in the biomedical domain, the complexity of the ontological axioms results in significantly large query expansions which present day query processing infrastructure cannot support. In particular, it remains unclear how to effectively parallelize such queries.

In this paper, we propose data and query transformations that enable inter-operator parallelism of ontological queries on Hadoop platforms. Our transformation techniques exploit ontological axioms, second order data types and operator rewritings to eliminate expensive query substructures for increased parallelizability. Comprehensive experiments conducted on benchmark datasets show up to $25 \mathrm{X}$ performance improvement over existing approaches.
\end{abstract}

\section{CCS CONCEPTS}

- Information systems $\rightarrow$ MapReduce-based systems.

\section{KEYWORDS}

Graph and network algorithms, improved models, algorithms, performance or scalability of specific applications and respective software, cloud workflow, data, and resource management including dynamic resource provisioning, data analytics and frameworks supporting data analytics, Scalable storage, metadata, namespaces, and data management

\section{ACM Reference Format:}

Hyeongsik Kim, Abhisha Bhattacharyya, and Kemafor Anyanwu. 2019 Semantic Query Transformations for Increased Parallelization in Distributed Knowledge Graph Query Processing. In The International Conference for High Performance Computing, Networking, Storage, and Analysis (SC '19),

\footnotetext{
${ }^{*}$ This work was done while the author was a $\mathrm{PhD}$ student at North Carolina State University

Permission to make digital or hard copies of all or part of this work for personal or classroom use is granted without fee provided that copies are not made or distributed for profit or commercial advantage and that copies bear this notice and the full citation on the first page. Copyrights for components of this work owned by others than the author(s) must be honored. Abstracting with credit is permitted. To copy otherwise, or republish, to post on servers or to redistribute to lists, requires prior specific permission and/or a fee. Request permissions from permissions@ acm.org.

SC '19, November 17-22, 2019, Denver, CO, USA

(C) 2019 Copyright held by the owner/author(s). Publication rights licensed to ACM.

ACM ISBN 978-x-xxxx-xxxx-x/YY/MM. . .\$15.00

https://doi.org/10.1145/3295500.3356212
}

November 17-22, 2019, Denver, CO, USA. ACM, New York, NY, USA, 13 pages. https://doi.org/10.1145/3295500.3356212

\section{INTRODUCTION}

A critical requirement for most present-day analytics tasks is to deal with multiple heterogeneous datasets. The issues of syntax and structural heterogeneities are typically dealt with using using ETL tools which are becoming increasingly more sophisticated. However, what remains an open challenge is effectively dealing with semantic heterogeneities where terms in different datasets are not reconcilable without significant human involvement, particularly when multiple domains are involved. The current trend for dealing with this problem with the use of formal ontologies, consist of machine-processible definitions of concepts and relations in a domain. These concepts and relations can then be used to annotate and link entities and concepts in different datasets. Their machineprocessible definitions enable automatic logical reasoning about relationships between data across datasets. This possibility offers two benefits: firstly, we can find more complete query answers and not require all facts to be explicitly represented and secondly, we are able to reconcile some semantic heterogeneities (both big wins for analytics in data integration scenarios).

Formal specification of such relationships has an impact on data analytics. Take for example a scenario where data (possibly warehoused from multiple sources) contain facts about entities Pers1, Pers2, suggesting the existence of two Persons that may be counted as two separate individuals during analytics tasks. However, imagine that we now have machine-processible predefined relationships such as motherOf, parent0f. If our dataset also now includes facts like: Pers1 motherOf Pers3; Pers2 parent0f Pers3; as well as formally defined concepts like Female with instantiation facts like Pers2 member Female. Finally, imagine that we have some ontological axioms: one that asserts that the relationship mother $0 f$ is a functional relationship (i.e. only one value possible); and one that asserts that motherOf is specialization of parentOf. Then, reasoners could automatically infer across these facts the conclusions: Pers2 motherOf Pers3; Pers1 = Pers2, avoiding the double counting. These machine-processible definitions of domain concepts, relations and associated axioms are what ontologies consist of.

W3C's Resource Description Framework (RDF) [7] is the common standard for representing ontological data. RDF's data model is a graph and the resulting models are often referred to as "Knowledge Graphs". Knowledge graphs have been very crucial to many lifesciences applications and other industry applications such as Enterprise/Web search. In-fact, the National Science Foundation recently launched a special program that has as one of its tracks 
building Open Knowledge Networks in line with its 10 Big Ideas. The goal of RDF is to facilitate automatic exchange, sharing and integration of data on the Web. Indeed, hundreds of such knowledge graphs have been curated by community efforts particularly in the LifeSciences [11, 23], and more generally in the Linked Open Data (LOD) cloud [8] which has publicly-available data from diverse domains and has grown exponentially over the past few years.

Processing ontological data or knowledge graphs requires the ability to handle explicitly represented facts captured in its graph structure as well as the ability to automatically infer implicit facts using ontological axioms. Ontological inferencing is computationally challenging as there are still open problems with scaling it up. As at the current time, knowledge graphs are typically manipulated using either graph engines $[17,24,43]$ or database engines. Database engines model data as ternary relationships and support pattern matching over such model in a manner similar to SQL. Knowledge graph processing in this approach results in many joins needed to reassemble related facts that are modeled using fine-grained ternary relations (unlike typical relational models where relations have a much higher arity). Graph engines depend on large amounts of main memory and tend to have scalability challenges and most focus only graphstructured processing and very limited ontological inferencing. Rule engines either (i.) first materialize all implied statements (i.e. a closed model) in a forward-chaining manner [29, 32, 41] which can then be followed by traditional query answering; (ii.) or use backwardchaining $[16,42]$ reasoners to derive only the implied facts that are relevant to a given query. Some hybrid approaches [16, 32] have also been proposed. However, these rule-based reasoning strategies tend to be inherently sequential, making them difficult to parallelize and scale up to Big Data and it is not obvious how to integrate traditional database query optimization techniques to improve them.

Recently, some alternative approaches in the spirit of database query optimization have emerged with a focus on specific classes of ontologies that are considered "first-order rewritable". These allow reasoning to be captured as first order logic formulae which can be expressed as relational query expressions. The excitement about this approach is that it essentially reduces the ontological inferencing to problems that comparatively mature database machinery can be applied to. More precisely, queries are written to Unions of Conjunctive Queries or (UCQs) [14] in which each union subquery essentially represents an alternative reasoning path for deriving implicit facts. For example, assuming the example scenario alluded to earlier, a query for all mothers would be expanded to (i.) any entities explicitly asserted as member0f Mother UNION (ii.) any entities that have at least one motherOf relationship with another entity UNION (iii.) any entities that are member female and have a parentOf relationship with another entity. By so doing, query processing handles both querying and ontological inferencing (but in a query driven way).

In theory, it should be possible to appropriate mature database query optimization techniques for improving efficiency of such queries. However, in practice, UCQs derived as ontological query rewritings are often orders of magnitude more complex than the heuristics used by traditional query optimizers can handle. It is not unusual for such queries to have up to hundreds, even thousands of union and join operators, whereas traditional optimizers typically $\overline{\text { scale only to about } \leq 20}$ join operations and disjunctive queries are also notoriously difficult to optimize. One important direction that has not been investigated thoroughly is: how parallelization can be used to scale up such "operator-dense" queries. In particular, given workloads with large number of partition-sensitive operations like joins that naturally result in lengthy execution workflows, how can inter-operator parallelism be enabled in parallel, shared-nothing computing environments like Hadoop[9] where only partitioned and intra-operator parallelism are the parallelization models? Addressing this challenge is the focus of this paper.

Contributions. In this paper, we present an approach that achieves our objective (albeit indirectly). Here inter-operator parallelism is achieved by transforming the semantics and behavior of operations and data in a way that allows partitioned parallelism computation models to still produce correct results but with much shorter, more efficient execution workflows. The proposed query and data transformation strategy combines multiple kinds of semantic transformations to achieve objectives including using specific ontological axioms to fold join sequences, factoring of subexpressions to the use of second order types and expressions. Specifically,

- A semantic reformulation technique that "folds" chain-joins into star-joins in a query (eliminating the need for a repartition step across joins).

- Reduce the size of query expressions by refactoring common subexpressions using novel adaptations of union-pushdown [15] and multi-query optimizations [30] query rewriting techniques to UCQs.

- Extend the query algebra for a second order type system for RDF (star subgraphs as first class entities) proposed in [26] with new operators and query rewriting rules that translations the Union operators in UCQs to disjunctions that can be used to extend filter predicates. Consequently, numerous Union operators are collapsed into filter predicates that can be evaluated concurrently, again eliminating cycles in execution workflow.

- An extensive evaluation conducted on synthetic datasets with customized query sets. The evaluation results show that our approach was up to $25 \mathrm{X}$ faster than existing approaches, using minimal footprint of disk/network I/Os.

The rest of the paper is organized as follows: Section 2 covers preliminaries, background and related work; Section 3 discusses our approach and its rationale; Section 4 reports on results of experimental evaluation.

\section{PRELIMINARIES}

\subsection{RDF/RDF(S) Data Model and Query Processing}

The RDF data model captures facts as triples which can also be viewed as graphs (each triple is equivalent to 〈node, edge, node $\rangle$ ). We will transition between the two data models in the paper depending on which better illustrates a point being made. In certain cases when query processing is being discussed, the triple relation view conveys the point better.

Fig 1 shows a collection of triples organized as a ternary relation (a very common data organization for database-style processing of RDF). The table shows two types of triples - data triples and schema triples. Data triples essentially capture instance data (assertions about 


\begin{tabular}{|c|c|c|c|c|c|c|}
\hline \multicolumn{2}{|c|}{ RDF Data Triples } & \multicolumn{2}{|c|}{ RDF Data Triples (Cont.) } & \multicolumn{3}{|c|}{ RDF Data Triples (Cont.) } \\
\hline :prs1 :affWith : & :u2 & :prs3 :affWith & :u2 & :FP1 & :researchInterest & "Research1" \\
\hline :prs1 :affWith : & :u3 & :prs3 :affWith & :u4 & :Dept1 & :name & "Dept1" \\
\hline :prs1 :hpage & "a.com" & :prs3 :affWith & :46 & :Dept1 & :subOrganizationOf & :Univ1 \\
\hline :prs1 :lectures & :crs1 & :prs3 :mbox & "c@g.c & : : 1 & :name & "Univ1" \\
\hline :prs1 :mbox & “a@g.com" & :prs3 :name & "c" & $:$ : 1 & rdf:type & :University \\
\hline :prs1 :name & "a" & :prs3 :name & "cc" & $\ldots$ & & \\
\hline prs1 rdf:type & :Faculty & :prs3 rdf:type & :Person & & RDF Schema Trip & iples \\
\hline :prs1 rdf:type & :Person & :prs3 rdf:type & :Faculty & :lectures & es rdfs:domain :Facult & \\
\hline :prs2 :affWith & :u1 & :crs1 :hpage & "crs1.edu" & :affWith & h rdfs:domain :Perso & \\
\hline :prs2 :affWith & : :43 & :crs1 :mbox & "crs1@n.edu" & :affWith & th rdfs:range $x$ sd:St & tring \\
\hline :prs2 :affWith & : u5 & :crs1 :name & "crs1" & :headof & of rdfs:subPropertyOf & f:worksFor \\
\hline :prs2 :mbox & "b@n.edu" & :crs1 :rating & $" 10 "$ & :worksF & For rdfs:subPropertyc & of :memberof \\
\hline :prs2 :name & "b" & :crs1 rdf:type & :Course & :worksF & For rdfs:domain :Emp & ployee \\
\hline :prs2 :name & "bb" & :FP1 :headOf & :Dept1 & :Employ & yee rdfs:subClassof : & :Person \\
\hline :prs2 rdf:type & :Person & :FP1 rdf:type & :FullProfessor & :worksF & For rdfs:range $\quad$ :Orga & sanization \\
\hline :prs2 rdf:type & :Faculty & :FP1 :name & "FProf1" & :subOrg & ganization Of rdfs:rang & ge:Organization \\
\hline
\end{tabular}

Figure 1: Example RDF Ontological Knowledge Base

instances of relationships or instances of class memberships). For example, the first row asserts that prs1 "is affliated With" an entity u2 (a university). The schema triples capture domain concepts and relationships and the relationships between them. Here we have domain concepts such as Faculty, Person and University and domain relations such as af $\mathrm{fWith}$ (representing an affiliation with an organization), headOf, lectures and sub0rganization0f and so on. There are some standard (RDF Schema RDF(S) [5]) metaclasses and metarelations used in defining domain vocabularies. A quick example is the metarelation rdfs: subclass0f which is used to assert for example that the class Employee "is a subclass of" the class Person. Note that the schema specification in figure is merely partial.

SPARQL [35] is the standard query language for RDF and its main query primitive is a graph pattern which itself is a set of triple patterns. A triple pattern is a triple in which components are variables (denoted with a leading ?) i.e. are not bound to constants. A triple pattern is matched against a data model based on its bound components. Then, parts of the matching triples that correspond to the triple variables are bound to the variables and are called "bindings" for that variable. For example, the first triple pattern shown in Fig 2 stp $_{1}\langle$ ?s, rdf:Type, ub:Student $\rangle$ has one variable?s in the subject position while $\mathrm{tp}_{4}$ in the second star pattern in the same figure has two variables ?c and ?n. The matches for the first triple pattern are all those triples that have property as $r \mathrm{df}$ : Type and $u b$ :Student as object. The bindings for the variable ?s are the subject components of all matching triples i.e. $\operatorname{stp}_{1}$ will match to the triple $\langle$ Student1, rdf:Type, ub:Student $\rangle$ where Student1 is the binding for the variable ?s.

When two triple patterns have a common variable that implies join between the bindings of the variables. For example, ?c variable is common between the two triple patterns $\operatorname{stp}_{1}$ and $\mathrm{stp}_{2}$ shown in Fig 3 . When the common variable occurs in subjects of multiple triple patterns (i.e. multiple subject-subject joins) we refer to that as a star join. When the common variable occurs in the subject and object (i.e. subject-object join) eg. the join between $s t p_{1}$ and $s t p_{2}$ in Fig 3 where the common variable is ?c which is the object for stp $_{1}$ and the subject in $\operatorname{stp}_{2}$, we refer to this as chain joins. It is very common to discuss SPARQL graph patterns in terms of star and chain patterns. For clarity, our example graph pattern is trying to find the list of students who have taken some courses.

To process graph pattern queries in a distributed setting, we partition the relation of triples on the join column. For this example, there are 4 join operations but only 3 data repartition steps are required: (i.) the partition on the ?s variable in the subject column which can support the 2 joins on that variable on that column, (ii.) the partition on the ?c in subject column needed for the 1 join on that variable and column (iii.) the join between the results of (i.) and (ii.) on the object column for the ?s variable and for the ?c variable on the subject column. The Fig 3(b) represents the same query as a graph. (i.) and (ii.) are equivalent to the two star joins and the chain join respectively. In general, any number of joins on a triple relation that form a star structure can be executed within the same partition phase. However, a star join and chain join cannot be executed in the same partition phase. Consequently, in a context where queries contain multiple star joins and chain joins, how to parallelize the execution of such queries is critical.

\subsection{RDF(S) Ontologies and Inferencing}

Using the RDFS standard metaclass rdfs: Class and metarelation rdfs: subClassOf we can define domain concepts e.g. in the academic domain example concept Faculty - $\langle$ Faculty, rdf : Type, rdf $\mathrm{s}: \mathrm{Class}\rangle$ states that Faculty is a class, and $\langle$ Faculty, rdfs:s ubClass0f, Person>. We can also define domain relationships e.g. worksFor, called properties in RDF parlance. A property is defined in terms of the types of entities it relates. The domain of a property defines which class instances a property can be applied to (the left side of relation) whereas the range defines which class instances can be values for a property (the right side). For example, based on the schema definition in the example in Fig 1, worksFor applies to concept Employee (domain) and can take values from concept Organization (range). RDF has a subsumption hierarchy not only on classes like in object oriented models, but also on relations. So for example, the relation headOf is a specialization of the worksFor relation and is stated as $\langle$ headOf, rdfs:subPropertyOf, worksFor

The RDFS standard also provides inferencing axioms which when combined with explicit data and schema triples allows for implied

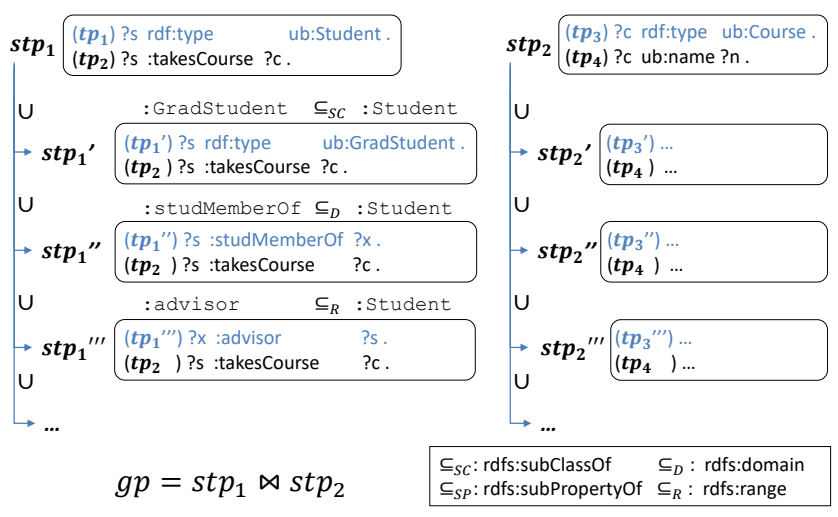

Figure 2: Rewriting two star pattern $\operatorname{stp}_{1}$ and $\operatorname{stp}_{2}$ 


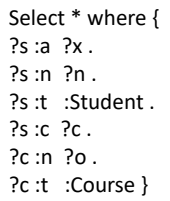

(a)

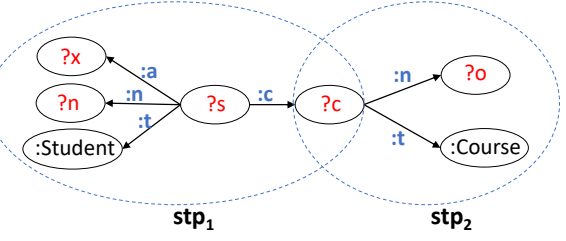

(b)
Figure 3: Example query graph pattern to find students taking courses (a) The query (b) The corresponding graph pattern

assertions to be derived. We illustrate a few axioms below (note consequents are above in this notation).

$$
\begin{gathered}
\frac{\langle ? c 1 \text { subClass } O f ? c 3\rangle}{\langle ? c 1 \text { subClassOf } ? c 2\rangle,\langle ? c 2 \text { subClassOf } ? c 3\rangle} \\
\frac{\langle ? \text { stypeOf } ? c 2\rangle}{\langle ? c 1 \text { subClassOf } ? c 2\rangle,\langle ? s \text { typeOf } ? c 1\rangle} \\
\frac{\langle ? s ? p 2 ? o\rangle}{\langle ? p 1 \text { subPropertyOf } ? p 2\rangle,\langle ? s ? p 1 ? o\rangle} \\
\frac{\langle ? \text { stypeOf } ? c\rangle}{\langle ? p \text { domain } ? c\rangle,\langle ? s ? p ? o\rangle} \\
\frac{\langle ? \text { otypeOf } ? c\rangle}{\langle ? p \text { range } ? c\rangle,\langle ? s ? p ? o\rangle}
\end{gathered}
$$

Rule 1a expresses the traditional transitivity or the subclass relationship (a similar rule exists for the subproperty relationship) essentially reasoning with only schema. Rule $1 \mathrm{~b}$ extends this reasoning to instances to say that if $c 1$ is a subclass of $c 2$ and a subject $s$ is a type of $c 1$, then we can infer that $s$ is also a type of $c 2$. Rule $1 \mathrm{~d}$ and 1e expresses inferencing based on domain and range relationships. $1 \mathrm{~d}$ says, if a property $\mathrm{p}$ has a concept $\mathrm{c}$ in its domain, and there is a statement that some subject $\mathrm{s}$ has property $\mathrm{p}$ with value $\mathrm{o}$, then we can infer that $s$ is of type $c$. The implication of rules like these is that you can derive data about type instances that are not explicitly stated. For example, although FP1 is not explicitly asserted to be of type Person, we can infer that from using rules $1 \mathrm{c}, 1 \mathrm{~d}$ and $1 \mathrm{~b}$ as shown below.

$$
\begin{gathered}
\frac{\langle F P 1 \text { worksFor Dept } 1\rangle}{\langle\text { headOf subPropertyOf worksFor }\rangle,\langle F P 1 \text { headOf Dept } 1\rangle} \\
\frac{\langle F P 1 \text { typeOf Employee }\rangle}{\langle\text { worksFordomain Employee }\rangle,\langle F P 1 \text { worksFor Dept } 1\rangle} \\
\frac{\langle F P 1 \text { typeOf Person }\rangle}{\langle\text { Employee subClassOf Person }\rangle,\langle F P 1 \text { typeOf Employee }\rangle}
\end{gathered}
$$

2.2.1 Ontological Inferencing in Query Processing. As alluded to in the introduction, to implement ontological inferencing as part of query processing, the idea is to rewrite a given query into an expanded UNION form that captures the inference paths needed to derive implicit facts. To see how this is done, take for example, a query with a triple pattern that matches the consequent of an inference axiom e.g. $\langle$ ?s, type $0 f, ? c\rangle$ in rule $1 \mathrm{~d}$. The rule will be used to expand the triple pattern with its precedents e.g. $\langle ? \mathrm{~s}$, type0f, ?c $\rangle:=\langle ? p$, domain, ?c $\rangle,\langle ? s, ? p, ? o\rangle$

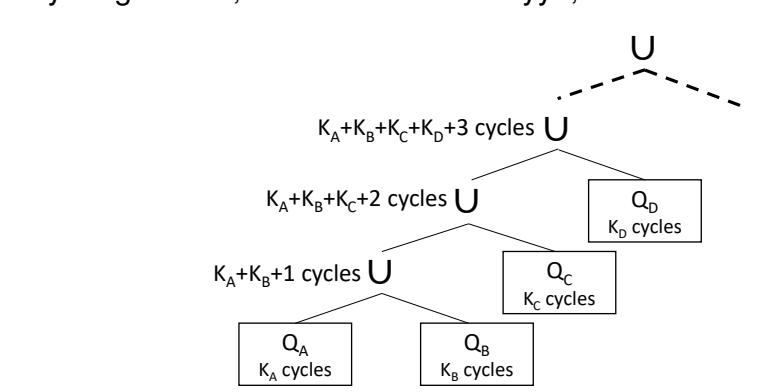

Figure 4: Example RDF Query plan

Fig 2 shows how the two star graph patterns $\operatorname{stp}_{1}$ and $\mathrm{stp}_{2}$ that are subqueries in our example query are expanded into $s t p_{1}^{\prime}$, $s t p_{1}^{\prime \prime}$, $s t p_{1}^{\prime \prime \prime}$ and $s t p_{2}^{\prime}$, stp $p_{2}^{\prime \prime}$, stp $p_{2}^{\prime \prime \prime}$ resp, as well as the rules used for each expansion. The completely expanded ontological query $q^{+}$is all the combinations of joins between the expanded subpatterns:

$$
\mathrm{gp}^{+}=\left(\operatorname{stp}_{1} \bowtie \operatorname{stp}_{2}\right) \cup\left(\operatorname{stp}_{1}^{\prime} \bowtie \operatorname{stp}_{2}\right) \cup\left(\operatorname{stp}_{1}^{\prime \prime} \bowtie \operatorname{stp}_{2}\right) \cup \ldots
$$

Fig 4 illustrates what a query plan structure for such queries would look like. It comprises of multiple subquery subtrees connected by union operations. The expanded query can be very large. For example, our simple example query find students enrolled in courses which is adapted from the LUBM benchmark ontology and dataset when expanded based on ontological axioms grows from 3 joins to more than 300 joins and hundred unions!!

This increased size has an implication for scalability and efficiency. For distributed processing, a primary concern is the number of phases (or repartition steps) in an execution workflow because these phases have a direct impact on cost of execution (in terms of network data transfer and I/O). Fig 5 shows what the execution workflow for our expanded query would be in a Hadoop processing context. The key thing to observe here is that given a seemingly trivial input query, after expansion we obtain an execution workflow with more than nine partition steps.

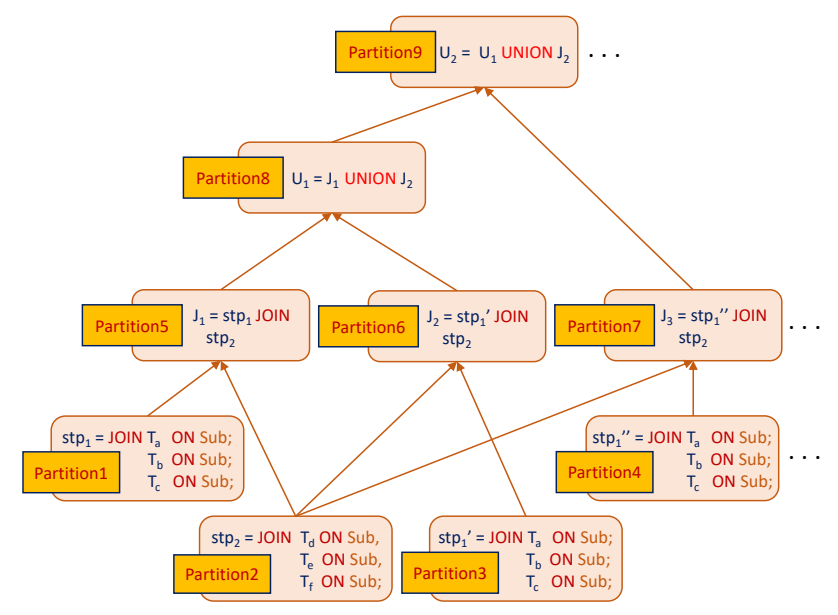

Figure 5: Figure showing partition steps in expanded ontological query 


\subsection{Reducing the Number of Query Operations in Ontological Query Rewritings}

2.3.1 RDF Data Storage Models and Query Processing. Fundamental to reducing the number of query operations is the issue of how data is modeled and represented. The two prevailing storage models for RDF are: (i.) triple-based storage models [19, 21, 31, 33, 39] which store individual triples independently and (ii.) subgraph-based storage models [34, 36, 44, 45] that prematerialize connected triples into coarser-grained units i.e subgraphs. The triple based storage models allow selective, fine-grained data access which has a benefit of reducing I/O as well as network data transfer in distributed processing. However, there is the overhead of requiring numerous expensive reassembly (join) operations for non-trivial queries. Some approaches that have been employed to mitigate some of this overhead include vertical partitioning - that partitions triples into equivalence classes based on their property types (edge labels) which reduces the likelihood of retrieving unwanted triples. In the context of distributed processing, partitioning techniques [18, 21, 31] have been employed to improve clustering of related triples, thereby increasing the possibility that most join operations can be done as local joins as opposed to expensive distributed join operations. On the other hand, these partitioning techniques are only beneficial to queries whose structures align with the partitioning strategy. For other scenarios, e.g. when query subgraph structures are larger than the partitioning units, co-location is no longer guaranteed and benefits are lost. Further, such techniques often increase the granularity of data access for simple operations adding unnecessary $\mathrm{I} / \mathrm{O}$ costs. Often, there is not a specialized index file organization used to serialize subgraphs which leads to having to do full data scans for most queries.

One consideration might be to adopt column-family stores such as BigTable $^{1}$ or its variations ( HBase $^{2}$ and Cassandra ${ }^{3}$ ) or (ii) columnar file formats such as ORC ${ }^{4}$ and Parquet ${ }^{5}$ for more efficient storage of the data units in the subgraph storage models. However, in big data contexts with a lot of heterogeneity (also encountered during our experiments with real data), the number of possible subgraph units exceeds what can be effectively supported by platforms like Hadoop due to the "many file/table/column family problem" 67 Further, some coarse-grained structures capture nesting which are not easily addressable using columnstores. Some other structures for multi-dimensional data such as [12,37] may at first appear useful for our context. However, users are still left with the challenge of being aware of what substructures exist in data for effective mapping to files.

2.3.2 Expanding Ontological Queries Only Partially. Efforts [40] to simplify ontological query rewritings to produce relatively less complex queries have proposed a technique that combines the rulebased inferencing with the query rewriting technique. The observation exploited is that by separating the expansions associated with generating implicit $r \mathrm{df}$ : type triples from inferencing that does

\footnotetext{
${ }^{1}$ https://cloud.google.com/bigtable

2 https://hbase.apache.org

${ }^{3}$ http://cassandra.apache.org

${ }^{4}$ https://orc.apache.org

5 https://parquet.apache.org

${ }^{6}$ https://cloud.google.com/bigtable/docs/schema-design

${ }^{7}$ https://groups.google.com/forum/\#!topic/parquet-dev/jj7TWPIUIYI
}

not involve $r d f$ : type statements but rather only schema assertions which are data-independent. As an example of reasoning only at the schema level, consider deriving all direct subClass $0 f$ relationships, thereby flattening a subClassOf hierarchy. Such reasoning can be done independent of the class instances i.e. the rdf : type statements. This results in a two-phase processing approach:

(1) Generate a partial closure materialization $\mathrm{kb}^{+\mathrm{S}}$ of a given knowledgebase $\mathrm{kb}$ that constitutes the given data triples plus only the closure over schema elements or the schema closure, that derives all non-rdf : type statements first.

(2) Then, given a query q, reformulate the query q into a union of graph patterns using a set of simplified inference rules that take into account (1.). The subqueries in the union queries include only expansions that derive implied rdf : type triples but none that do schema-only inference since the latter are precomputed in (1.) We denote the resulting query as $q^{\text {ref- }}$. (Since ( $q^{\text {ref- }}$ encodes fewer inferencing paths, the resulting reformulated queries are naturally smaller and less complex i.e. have smaller union width). Note that output of (1.) can be reused for any query.

(3) Evaluate the reformulated query $\left(q^{r e f-}\right)$ over $\mathrm{kb}^{+\mathrm{S}}$, to produce the complete set answers $q^{r e f-}\left(\mathrm{kb}^{+}\right)=q\left(\mathrm{~kb}^{\infty}\right)$.

Issues. While this approach enables a reduction in the number of query operators, the resulting reduced query expressions are still large. Therefore, this approach represents a step in the right direction and should be built on, but does not completely solve the problem.

2.3.3 Reducing the Number of Query Operations Using Common Subexpression Factorization. Observe that the non-expanded triple patterns such as $t \mathrm{p}_{2}$ and $t \mathrm{p}_{4}$ need to be included in every union subquery. Further, all possible join combinations of expanded subpatterns $\operatorname{stp}_{1_{i}}$ and $\operatorname{stp}_{2}$ need to be included in the overall expanded query. Consequently, ontologically rewritten UCQs often have a nontrivial amount of commonality in subexpressions across subqueries. The following techniques from relational query processing may be adapted for eliminating common subexpressions.

Rewriting Using Multi-Query Optimizations Although multiquery optimization techniques (MQO) are designed for sharing interquery common subqueries, they can be adapted for intra-query optimizations for UCQs because we can temporarily handle the different subqueries as different queries and then merge later on. MQO technique for SPARQL queries have been proposed in [30] which uses a left outer join operator to link the common subexpression to the different remaining subexpressions. This translation leads to a relaxation of the semantics of the query and requires a post processing step to filter out results that are invalid with respect to original query semantics.

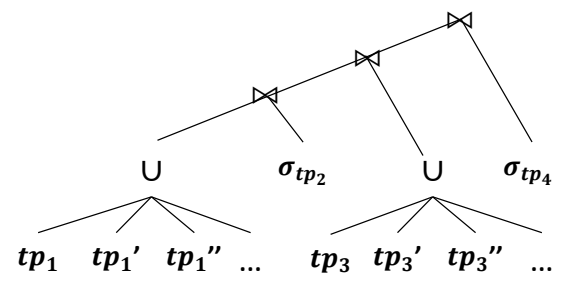

Figure 6: Execution plan showing unions under joins to avoid repeated evaluation of common subexpressions 
Rewriting Using Union-Push-Down The second strategy is to adapt union-pushdown techniques to push unions under joins to avoid repeated evaluation of common subexpressions. Fig. 6 shows the query plan based on this approach. Triples matching $t p_{1}$ and all of its reformulations such as $t p_{1}^{\prime}$ and $t p_{1}^{\prime \prime}$ are first merged using unions and then joined with other triple patterns. $t \mathrm{p}_{3}$ and its reformulation is similarly processed. The potential benefits of this strategy could be several: in addition to avoiding repeated evaluations of common expressions, this technique significantly simplifies the plan, reducing the overall number of operators and re-partitions of intermediate data.

Limitations. For the MQO techniques that replace unions with outer joins, in addition to the additional postprocessing overhead to correct semantics of result, the intermediate results produced before postprocessing could be quite large which can present resource limit challenges in practice [1,2]. For the Union-Push-Down technique which first merges all triples matching triple patterns using unions before pruning out irrelevant triples using joins, the size of input of the join is also very large and increases the cost of shuffling and sorting such data across the network for parallelized join processing.

\section{OPTIMIZING UCQS USING TYPE-BASED SEMANTIC REFORMULATIONS}

One potentially interesting idea for enabling more parallelism in processing of UCQs is to reinterpret the unions as disjunctions. To use a simple example on a relational table about Boats. If we want to find all boats colored red or green, we can either have a two subqueries (one for each color) and then union them or we can apply a disjunction with filter predicate filter(color = 'red' OR color $=$ 'green'). The advantage here is that while the union query comprises two filter subqueries and a union i.e 3 operations, the filter with disjunction processes multiple subconditions in a single operation. However, to adapt this idea to UCQ processing, we would need higher order filter predicates whereas the relational example is first order (operations on sets). The reason is that the set of alternatives that we consider here are graph patterns and star subgraphs which are sets of sets of triples and triple patterns. In

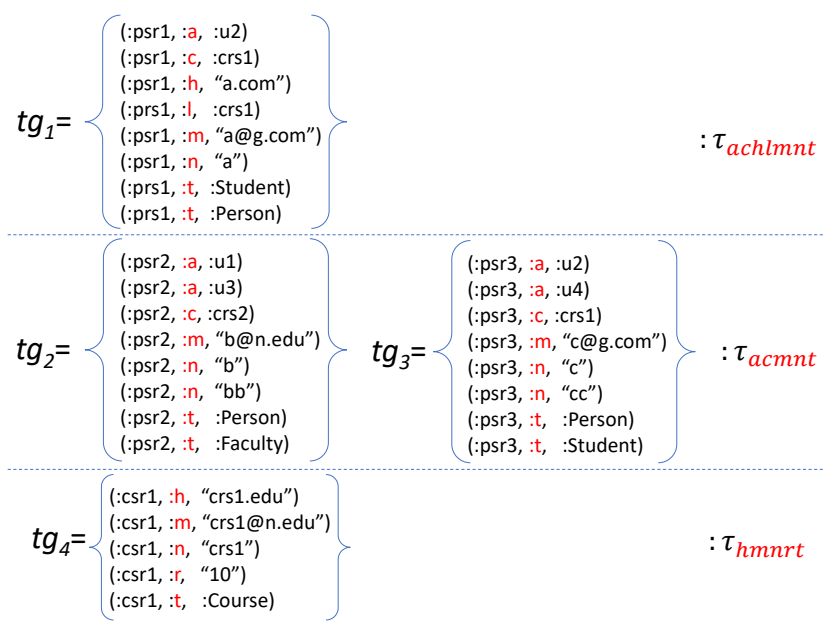

Figure 7: Figure showing example triplegroups and corresponding R-Type short, we would need to make "sets of sets of triples" first class citizens in our data and query model and enable query operators to process them as such. Fig. 7 shows our original example partitioned into equivalence classes based on the types of properties that a subject entity has. For example, $\operatorname{tg}_{1}$ groups all the triples for subject :prs1. This is called a triplegroup in the parlance of $[25,36]$. Some triplegroups may be similar or equivalent in terms of what properties they have e.g $\operatorname{tg}_{2}$ and $\operatorname{tg}_{3}$ which have the same set of distinct property types a, m, n, t. On closer observation, it easy to see the correspondence between star graph patterns and triplegroups. This relationship makes it possible for a star graph pattern in a query to be evaluated simply by matching it against a triplegroup equivalence class that comprises the same properties as that in the star pattern query.

Some preliminary work [3] interprets these equivalence classes as types and builds a type system for RDF called R-Types. For example, $\tau_{a m n t}$ is a type whose interpretation is the set of triplegroups with same subject, and all properties for that subject is one of a, $m, n$, t. Beyond the typing system, a Hadoop-based indexed file storage for efficient storage and retrieval of $R$-Types has been proposed by [27]. Some complimentary work on developing a query algebra for manipulating triplegroups called the Nested TripleGroup Algebra (NTGA) has been proposed in [25, 36] .

Two core NTGA query operators are : (i) TG_TypeSelection $\left(\sigma^{\gamma}\right)$ that applies a meta-query that computes relevant type names and (ii) TG_Projection $\left(\sigma^{\pi}\right)$ that projects relevant subgroups of triples from type-matching triplegroups. To adapt these operators to our problem, we need to generalize the previous operators to process predicates containing disjunctions over star subgraphs. In other words, enabling query expressions such as: TG_TypeSelection $\left(\sigma^{\gamma}\right)$ to be generalized to handle disjunctions. Conceptually, this would allow our example graph pattern gp to be transformed into a disjunctive expression such as:

$$
\mathrm{gp}^{\prime}=\sigma_{\left\{\operatorname{stp}_{1} \vee \operatorname{stp}_{1}^{\prime} \vee \ldots\right\}}^{\bowtie^{\gamma+}} \sigma_{\left\{\operatorname{stp}_{2} \vee \operatorname{stp}_{2}^{\prime} \vee \ldots\right\}}^{\gamma+}
$$

where (i) $\sigma^{\gamma+}$ selects all star subgraphs based on the star patterns in the query and its expansions and (ii) $\bowtie^{\gamma+}$ joins relevant subgraphs. We propose the operator extensions Multi-TypeSelection $\left(\pi^{\gamma}+\right)$ and Multi-Projection $\left(\sigma^{\gamma}+\right)$ to select type names matching multiple type signatures and project out irrelevant triples among different typed triple sets respectively.

\subsubsection{Implementing Extended R-Type Query Operators for} UCQs. Current NTGA query operator implementation accepts single filter conditions. To implement these generalized operators, we need some helper functions. Let $\{s t p\}^{+}$be defined as the set of all possible reformulations of star pattern stp and $\operatorname{propset}\left(\{s t p\}^{+}\right)$ also be a function such that propset $\left(\{s t p\}^{+}\right)$returns sets of Properties in reformulated star patterns, $\operatorname{propset}\left(\{s t p\}^{+}\right)=\left\{\operatorname{prop}\left(s t p_{1}\right)\right.$, $\left.\operatorname{prop}\left(s t p_{2}\right), \ldots, \operatorname{prop}\left(s t p_{m}\right)\right\}$ where prop(stp) returns the distinct property types in $s t p$. The interpretation of reformulated or expanded star patterns $\{s t p\}^{+}$(or $\left[\left[\{s t p\}^{+}\right]\right]$) can then be expressed in terms of the existing operators with extended input parameters as follows.

$$
\left[\left[\{s t p\}^{+}\right]\right]=\pi_{\text {propset }\left(\{\operatorname{stp}\}^{+}\right.}^{\gamma+}\left(\operatorname{sig}^{-1}\left(\sigma_{\text {propset }\left(\{s t p\}^{+}\right)}^{\gamma+}(P N)\right)\right)
$$

Note that unions in UCQs are pushed down and implicitly captured as disjunctions in the filter predicate, which allows reducing 
the number of joins and repartitions steps to 1 . Thus due to the introduction of these new operators the resulting execution workflow is much shorter.

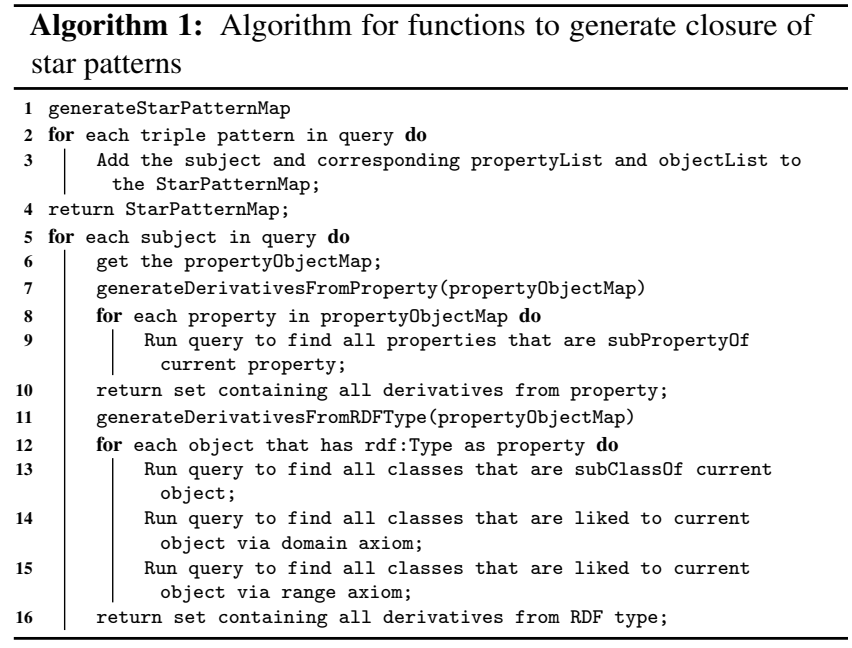

Let us consider the previously mentioned example query to find the the list of students who takes some courses and the triplegroups shown in Fig 7 as the data. The query has two distinct star patterns stp $_{1}$ where the subject variable is ?s and stp $_{2}$ where the subject variable is ?c. Two helper functions generateDerivativesFrom Property and generateDerivativesFromRDFType are used find the closure of the star patterns or $\{s t p\}^{+}$be defined as the set of all possible reformulations of star pattern stp. To use these functions a starPatternMap must be first generated. This is done by line 2-4 in Algorithm 1 where we iterate through each triple pattern in the query and add the subject, list pf property and list of objects to a map datastructure. For our example query the starPatternMap should be $? \mathrm{~s}=[\mathrm{a}=[? \mathrm{x}=], \mathrm{n}=[? \mathrm{n}], \mathrm{t}=[$ Student $], \mathrm{c}=[? \mathrm{c}]], \mathrm{c}=[\mathrm{n}=[? \mathrm{o}]$, $\mathrm{t}=[$ Course $]$.

Now for each subject in the starPatternMap we must run the two helper functions to get the full closure of that star pattern. We fist get the propertyObjectMap for each subject in line 6 of the Algorithm 1 and then call generateDerivativesFromProperty in line 7. This function iterates through each property (except for property $\mathrm{rdf}$ : Type) and fins all properties in the ontology which have a subProperty0f relation with it. Suppose the ontology has a triple $: b$ rdfs:subPropertyOf $: a$ then $b$ will be added to the derivatives set in line 9 .

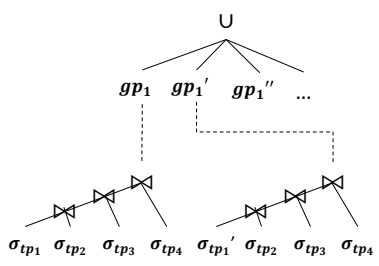

(a)

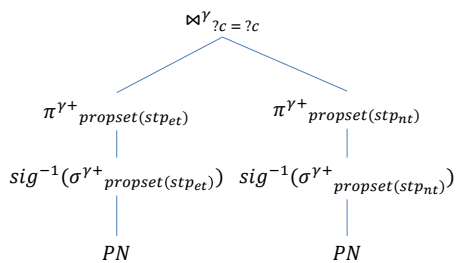

(b)
Figure 8: Interpreting ontological query gp as (a) unions of FOL formulas and (b) second order filter conditions with disjunctions
For the rdf : Type triples, we must call generateDerivatives FromRDFType function which iterates through all objects that have rdf : Type as its property in lines 12-15. If there exists triples such as GradStudent rdfs:subClassOf Student then this is also added to the derivatives set. In this way the two functions help build the full closure set of all star patterns in the given query.

Fig. 8(b) shows the difference in execution plans for gp. In Fig. 8(b) propset $\left(\left\{s t p_{e t}\right\}^{+}\right)$in the left subplan returns (i) the properties of $s t p_{e t},\{$ :takesCourse, rdf:type $\}$, and (ii) the properties of the reformulated star patterns, e.g., \{:takesCourse, :studMemberOf $\}$ from $s t p_{e s}$. Applying $\sigma^{\gamma+}$ returns all type names relevant to these star patterns, e.g., $\tau_{e t}, \tau_{\text {est }}, \tau_{\text {estp }}$, etc. $\pi^{\gamma+}$ then selects triplegroups of relevant types and eliminate irrelevant triples in these triplegroups, returning triplegroups that exactly match $s t p_{e t}$ and its reformulations. The right subplan is similar. Note that (i) this plan consists of only 5 operations and requires 1 data repartition for joining relevant triplegroups and is less sensitive to the complexity of ontologies while the relational style UCQ plan in Fig. 8(a) involves 400 selections and 300 joins leading to too many data repartition steps. A final comment regarding this rewriting strategy is that the ability to make multiple star join subqueries reduce to a single filter operation (albeit with multiple disjunctions) is that star joins are data partitioning equivalent. This is so because although different star joins are evaluating different join variables, the joins are all happening on the same column in the relation (usually the subject column) so that data partitioned on that column can be used to evaluate the different star subquery patterns.

\subsection{Rewriting Techniques for Eliminating Chain-Joins via Inverting}

While the rewriting approach based on disjunctions is based on star subgraphs and star joins, the reality is that graph pattern queries typically have both chain and star joins. Further, some of the rewriting rules for ontological inference inherently introduce chains. Fig. 10(b) shows that the original query $Q$ consists of a single star pattern with an $r d f$ : type triple pattern, but rewriting this query using R4 and $\mathcal{D}$ leads to the graph pattern $Q^{\prime}$ that consists of the two star patterns connected by a chain-join via the variable ?c, e.g., $\langle$ ?s; $\mathrm{ub}$ : teacher0f ; ?c $\rangle$ and $\langle$ ?c; ub: name; 'Course0' $\rangle$.

Unfortunately, chain-joins are not data partition equivalent with star joins. Consequently, a repartition step would be necessary. However, we can eliminate chain joins by collapsing them into star joins using the fact the properties or binary relations have inverses that can substituted to flip chain joins. This is particularly reasonable when we observe that an inverse triple pattern is not connected with other triple patterns except its chain-join, i.e. the Subject variable of the inverse triple pattern is not overlapped with other variables in a query. For example, $\langle$ ?s ub: teacher $0 f$ ?c $\rangle$ in $Q^{\prime}$ can be inverted into $\left\langle\right.$ ?c ub:teacher $0 f^{-1}$ ?s $\rangle$ which transforms the chain-join into the star join as shown in Fig. 10(b). However, simply inverting triple patterns would not be sufficient due to a number of issues, e.g., (i) inverting triple patterns would result in changing semantics of queries and (ii) inverted triple patterns need to be differentiated with original triple patterns to avoid inadvertently adding superfluous triple patterns in queries. 


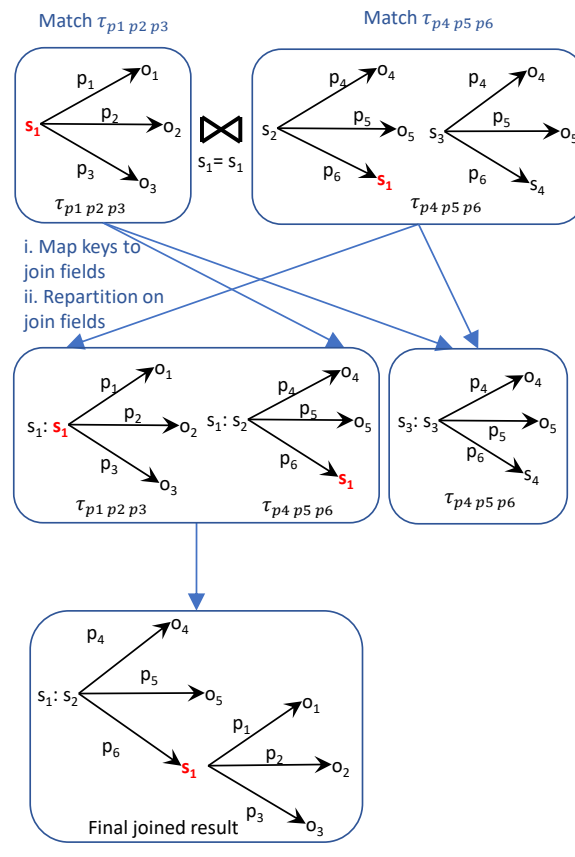

(a)

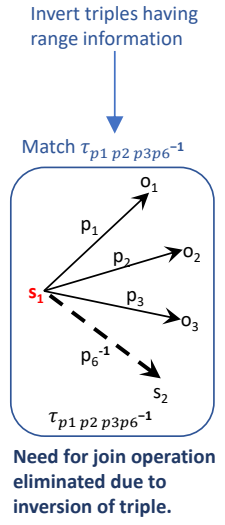

(b)

Figure 9: (a) Query processing using relational join approach (b) Eliminating join due to inverse expression

To address these issues, we propose to rewrite both query and data. Specifically, we introduce inverted triples matching inverse triple patterns and annotate inverse triple (patterns) with special markers to denote that they are inverse ones. The concept of the inverting can be formalized as follows.

Definition 3.1. (Inverting Triples) Let $H$ be a set of schema triples and $\mathrm{t}_{r}$ be a triple of the form $\langle\mathrm{s}, \mathrm{p}, \mathrm{o}\rangle . \mathrm{t}_{r}$ is defined as rangeinvertable triple if ( $\mathrm{p}, \mathrm{rdfs}$ : range, $\mathrm{c}) \in H$. Inverting $\mathrm{t}_{r}$ forms a new inverted triple $t_{r}^{-1}$ with the annotated Property $\mathrm{p}^{-1}$, i.e., $\langle$ o, $\left.\mathrm{p}^{-1}, \mathrm{~s}\right\rangle$.

Example 3.1. (Generating Inverse Triples) Fig. 10(a) shows that the triple $\langle\mathrm{ub}:$ prof 1 , $u b:$ teacher $0 \mathrm{f}, \mathrm{ub}: \mathrm{c} 1\rangle$ is the range-invertable triple because the ontology 0 contains $r \mathrm{df} \mathrm{s}$ : range axiom for the Property ub: teacher $0 f$. Thus we generate an inverse triple $\langle u b: c 1$ $\mathrm{ub}:$ teacher $\left.0 \mathrm{f}^{-1}, \mathrm{ub}: \operatorname{prof} 1\right\rangle$ and further leads to the generation of two triplegroups typed as $\tau_{t} \ldots$ and $\tau_{t^{-1} n \ldots}$ ( $\mathrm{t}$ and $\mathrm{n}$ are abbreviations of ub: teacherOf and ub:name).

As a final note, range-invertable triples introduced by semantic optimizations do not produce any superfluous results which can be proved as follows.

LEMMA 3.1. Introducing range-invertable triples does generate spurious query answers.

PROOF. The set of properties used for range-invertable triples are disjoint from the original set of properties. Consequently, there is no possibility of ambiguity in matching query triple patterns By inverting both triple patterns and related data, the equivalence between triple patterns and matching triples is maintained and will

not lead to lossiness. Finally, the corresponding between rewritten triple pattern and the other triple patterns that it is supposed to be joined with is maintained. The only change is that the join column changes.

\section{EMPIRICAL EVALUATION}

\subsection{Testbed Setup}

The goal of our evaluation was to compare the performance of our approach with other rewriting techniques for UCQs. We tested three cases using five different types of rewritings including ours. The first case was varying the size of rewritings (union branches), the second one was varying the size of common expressions, and the third one was varying the size of axioms for testing the effectiveness of semantic optimizations.

Evaluated Approaches. We extended type-based query processing system in [28] to include query rewriting techniques and semantic optimizations presented in this paper and denoted it as SemStorm+. To evaluate and compare, we considered employing recent Hadoop-based RDF query processing systems [6, 18, 22, 31, 33, 38, 44]. However, none of them fully support 'all' constructs such as FILTER, OPTIONAL, and UNION. Apache Hive(1.2.2) supports the individual operators but does not have the query rewriting rules needed in its optimizer. Therefore, we bypass the query rewriting phase for Hive by directly providing manually rewritten queries in HiveQL. The queries are equivalent to what would have been produced if Hive's optimizer employed the rewriting techniques discussed in Section 2.3. The final SPARQL and corresponding HiveQL queries are available in the project webpage at [4].

(1) Hive(U) simulated a default query rewriting that generates UCQs.

(2) Hive(M) rewrote UCQs using MQOs [30] i.e. non-overlapping patterns in union branches are mapped into OPTIONAL clauses. Since HiveQL does not support these, we use left outer joins to simulate the semantics of OPTIONAL constructs. We also inserted a post-processing operation that (i) extracts triples matching each subqueries from the result of left outer joins and (ii) merge them using unions.

(3) Hive(N) reformulated UCQs using union pushdowns, i.e. triple patterns and their rewritings are nested (grouped as subqueries) and joined with other triple patterns.

(4) Hive(D) transformed UCQs as a basic graph pattern matching query with disjunctive predicates and objects.

To simulate these approaches, RDF triples were loaded into HDFS and imported into a ternary table $T$. As optimizations, all approaches except the Hive $(D)$ employed vertical partitioning, which partitions table $T$ partitioned based on Property types using a map-only job per Property type. The reformulation technique for eliminating schema triple patterns are also applied to all other approaches so that the number of union branches to be evaluated can be equal for all. We also considered evaluating centralized systems that use query reformulation techniques for logical inferencing $[10,16]$ but they were excluded because only limited entailment rules were supported or the reasoning result was incomplete as discussed in [13]. All tested queries and details are available at our project site [3].

Benchmark Dataset and Queries. Our testbed consists of Lehigh University Benchmark (LUBM) [20] benchmark datasets, which are synthesized based on an ontology from academic domain. We 


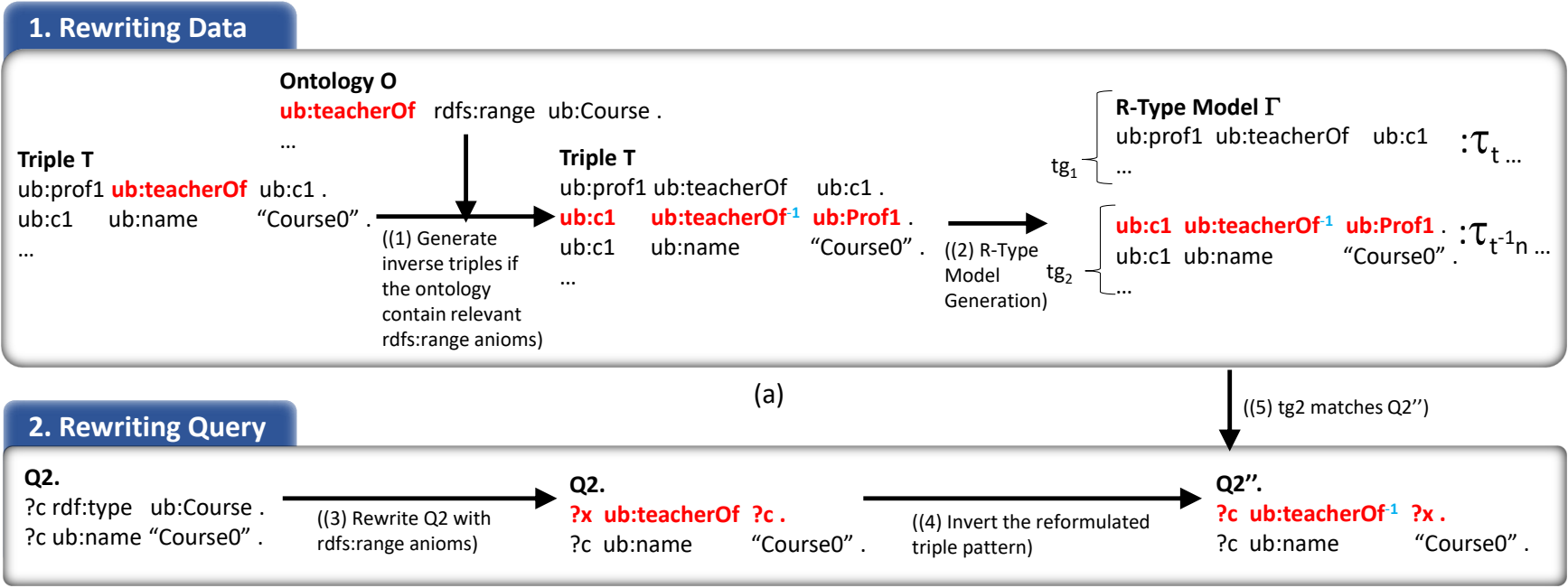

(b)

Figure 10: An overall flow of semantic optimizations for range-invertable triples: (a) data and (b) query rewriting process.

employed LUBM $1 k$, LUBM datasets with $1 k$ university $(23.8 \mathrm{~GB}$, $138 \mathrm{M}$ Triples) and LUBM $2 k$, LUBM dataset with $2 k$ university (47.9GB, 267M Triples). We tried larger datasets LUBM $5 k, 10 k$, or $20 k$ but found that most approaches failed due to the lack of resources such as memory or not being able to produce results in two hours even for highly selective queries. Therefore, we report experiments for only the dataset $(1 k)$ for which all approaches produce results. For $2 k$ we provide some results that give insight into the behaviour of the different approaches.

Testbed queries were adapted from LUBM benchmark queries to incorporate scenarios on varying characteristics of UCQs such as sharing the evaluation of common expressions and the impact of semantic optimizations. Query selectivities were also varied by binding Object fields of triple patterns (high-selectivity) and evaluating same query with unbound Object (low selectivity). We observe that the execution of some queries required significantly longer time than others, thus we set a timeout period, i.e., we aborted the queries a specified time period (1 hour) and marked such queries using a blue tilde. Approaches that failed to produce answers due to errors such as job failures, are marked using a red $\times$. Due to space constraints, we present only results with high-selective queries here.

Cluster Configuration. Evaluation was conducted on 45-node Hadoop clusters in university's virtual computing lab, with each node equipped with Xeon dual core x86 CPU (2.33 GHz), 4GB RAM, and 40GB HDD. While cluster sizes may appear larger than necessary, the 45-node cluster made available 900GB (20GB per node) which was required to process and store large intermediate data generated while processing UCQs. All results were averaged over three or more trials.

\subsection{Evaluation Results}

4.2.1 Pre-processing Input Datasets. Hive $(D)$ used a ternary relation $T$ without any extra preprocessing thus its preprocessing time was the shortest but its storage requirement was higher than others. Other Hive-based approaches used the VP approach thus its preprocessing time was longer but space requirement was low due to removal of repeated Properties. SemStorm + required the least amount of space because of its efficient storage model, e.g., common nodes across triples in triplegroups are only stored once. We also measured the requirement of space and execution time for materializing types generated by inverting triples. Among 31 property types, 18 property types of LUBM has relevant $r d f s$ : range axioms, which takes up approx. $23 \%$ of datasets. However, only approx. $8 \%$ and $5 \%$ of additional space and time were required because of the efficiency of SemStorm+'s storage model. Fig. 11 shows results of space requirement and pre-processing time for the LUBM $1 k$ and $2 k$ datasets. Additional details on the storage requirement are available on the project website [3].

4.2.2 Varying the Number of Union Branches. In this task, we varied the number of union branches by adjusting the number of relevant axioms and studied its impact. We began with Query $A$, which is a single triple pattern with Property rdf:type and Object ub:Person. We then adjusted the number of axioms so that the query can be expanded from 0 to 5 union branches ( $A 1$ to $A 6$ ). Query $B$ and $C$ are built on $A$ but additionally include a single nonexpandable triple pattern that shares the same subject variable but had different selectivities. Query $D$ included the second expandable triple pattern with Property rdf:type and Object ub:Publication which was chain-joined with $A$ via a single non-expandable triple pattern. We fixed the number of union branches for ub:Publication ( 8 branches) and increased the number of branches for ub:Person

\begin{tabular}{|c|c|c|c|c|}
\hline \multirow{2}{*}{ Raw } & \multicolumn{3}{|c|}{ Storage Requirement (GB) } & \multicolumn{2}{c|}{ Pre-processing time (secs) } \\
\cline { 2 - 5 } & LUBM 1K & LUBM 2K & LUBM 1K & LUBM 2K \\
\hline Hive(U, N, M) & 23.8 & 47.9 & - & - \\
\hline Hive (D) & 13.7 & 27.7 & 1367.62 & 1584.36 \\
\hline Semstorm+ & 17.5 & 35.2 & 146 & 198.12 \\
\hline
\end{tabular}

Figure 11: Storage requirement and preprocessing time of the benchmarked approaches 


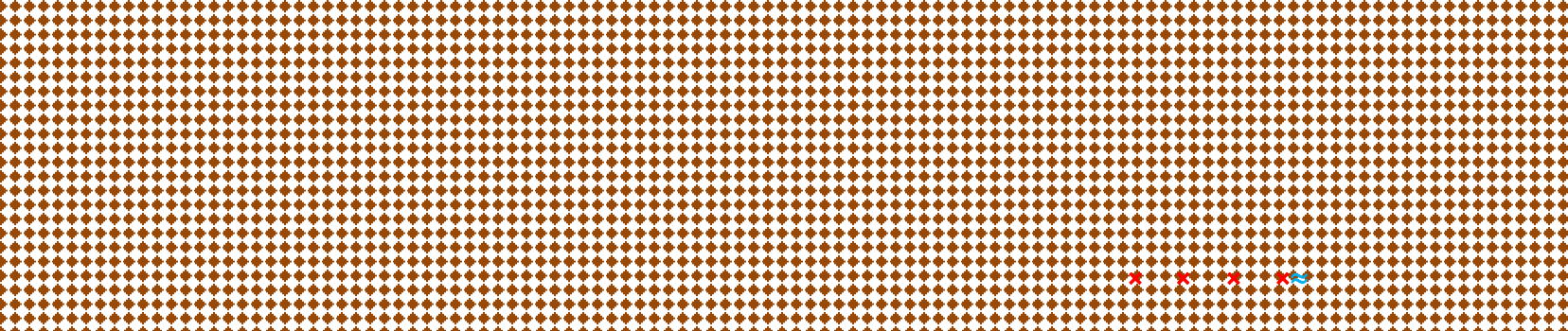

Figure 12: Execution time of queries with a varying number of union branches (unit: s)

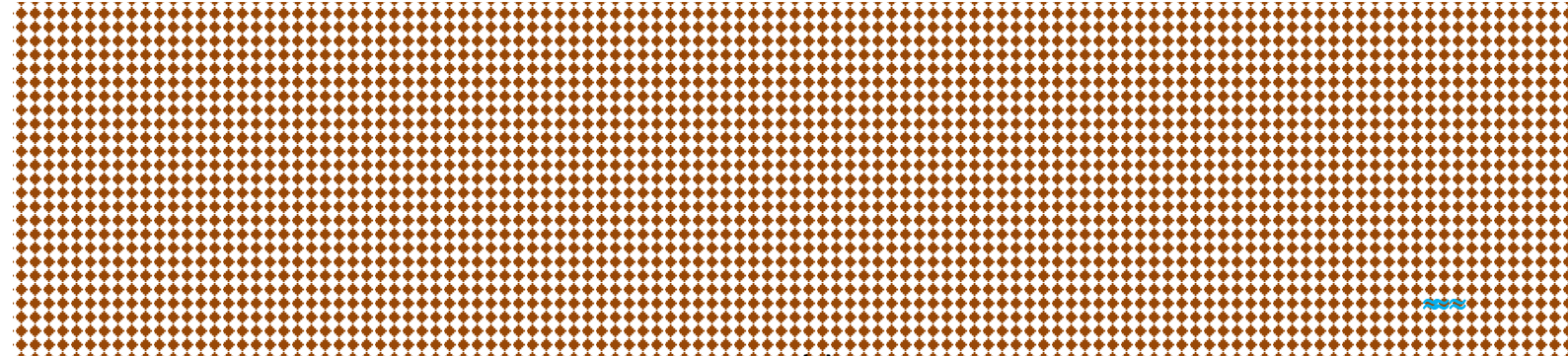

Figure 13: Execution time of queries with a varying size of common expressions (unit: s)

from 1 to 4, generating four queries (D1-D4) that have 8,16, 24, and 32 of branches. Query $E$ was similarly constructed but it included additional non-expandable triple patterns. Execution times of the tested queries are shown in Fig. 12.

Hive $(U)$ processed every union branch at the same time to maximize the parallelism, which worked in a small number of union branches. However, its execution time rapidly increased as the number of branches increased, e.g., the execution time of E1-E4 where \#branches increased from 8 to 32. Adding union branches led to adding a large number of selections and joins in the plans, causing overhead to (i) the application master node that manages the execution of query plan and (ii) the slave nodes that executes actual operators. For example, E4 is translated to approx. 500 operators. Hive also sometimes cannot generate plans for queries such as $D 1$ $D 4$, thus failed to process such queries. In $\operatorname{Hive}(D)$, its execution time also became longer as the number of filter conditions (union branches) increased because a single triple needs to be evaluated over many filter conditions, delaying scanning process. This issue became worse as the number of triple patterns increased because this approach had to scan a relation $T$ multiple times for every combination of filters in queries (e.g., E1-E4).

Adding new union branches in Hive (N) only added selection operators without any join operators, thus this approach was better than Hive $(U)$. However, its performance was not far better because this approach also had to transfer all triples matching union branches for joins, while many of transferred tuples would likely be irrelevant and later filtered out as a result of joins. The number of such triples increased fast in queries such as D1-D4 and E1-E4) and therefore the execution time significantly increased. Hive $(M)$ became significantly slower as the number of branches increased because the size of intermediate results rapidly increased as the number of left outer joins increased. This significantly impacted the performance in distributed environments because a huge amount of intermediate data needs to be shuffled and sorted for join operations. This issue was particularly severe when the number of branches(optional clauses) increased fast such as E1-E4, causing failures during query processing due to the OOM. In SemStorm+, the execution time was also increased but its rate was extremely low because, unlike other approaches, adding union branches only led to the increment of matching types while the overall structure of query processing plan is maintained without increasing the number of operators. Therefore, even for complex queries such as E1-E4, the performance of SemStorm + was relatively stable and much faster than others.

4.2.3 Varying the Size of Common Subexpressions. This task studied the impact of the size of common subexpressions in UCQs. For this study, we built a custom query set where query $F 1$ consists of a single triple pattern with Property rdf:type and Object ub:Person which is expanded into 14 different triple patterns. Other queries include additional non-expanding triple patterns. F2-F4 included the single non-expanding triple pattern with three different Properties that show decreasing selectivity (increasing popularity). Similarly, $F 5-F 7$ included the two non-expanding triple patterns which use the same set of properties in $F 2-F 4$. Similarly, $F 8$ contains three nonexpanding triple patterns. We also tested other class in ontology, i.e.,
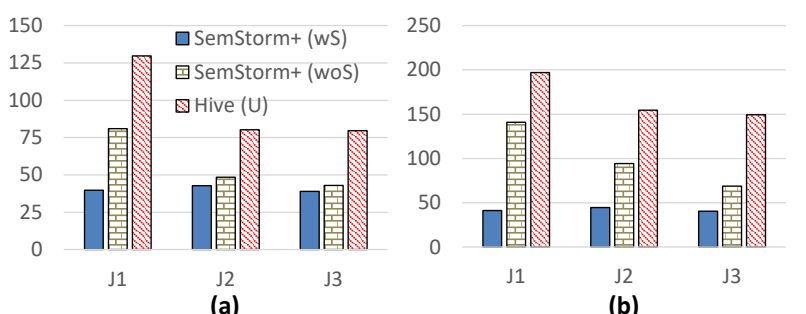

Figure 14: Execution time of queries with varying number of rdf s : range axioms: (a) high and (b) low selectivities (unit:s) 


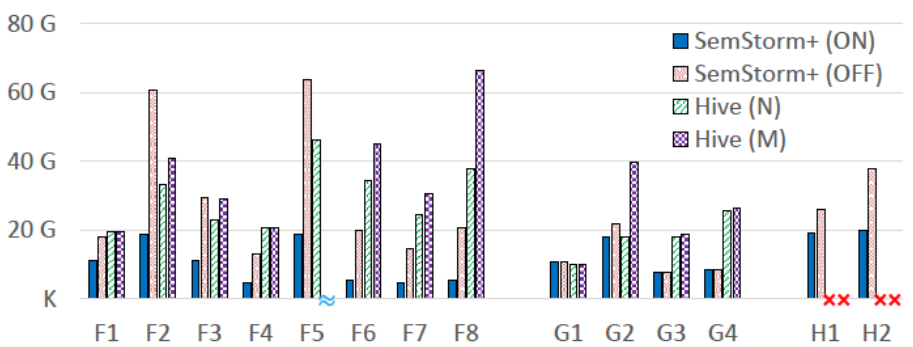

(a)

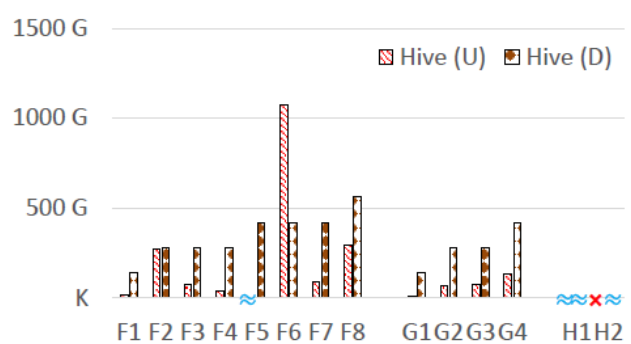

(b)

Figure 15: HDFS read of queries with a varying size of common expressions: (a) high and (b) low selectivities (unit: GB)

G1 was similar to $F 1$ but used ub:Publication as Object, which produced 8 different rewritings. We similarly designed the query G2-G3 and $G 4$ to include the single and the double non-expanding triple patterns. Query $\mathrm{Hl}$ was the two star pattern query built by combining $F 1$ and $G 1$. $H 2$ is similarly built but additional non-expanding triple patterns are included for each star pattern. Execution times for different approaches are shown in Fig. 13.

In Hive $(U)$, the same vertical partitions were repeatedly processed for common subexpressions. This execution strategy therefore led to the excessive uses of task slots and I/Os at the same time, slowing down overall performance such as $F 2, F 5, F 6$ and H1. Hive(D), Hive $(N)$, and Hive $(M)$ generally showed better performances because these approaches allowed sharing the evaluation of common expressions. However, as the selectivity of common expressions becomes lower, the size of intermediate data matching common subexpressions rapidly increased, while a large amount of data is irrelevant, which was filtered out using joins. This process involved a substantial disk and network I/Os. These approaches often resulted in timeouts or failures (e.g., $\mathrm{H} 1$ and $\mathrm{H} 2$ ) in the end. SemStorm+ showed the best performance particularly over queries with lowselective common subexpressions such as queries $F 2, F 6$, and all $H$ queries because SemStorm + was able to filter out irrelevant types as early as possible while the processing of relevant types for common subexpressions was shared. To measure the effectiveness of sharing scans, we tested the case that disables the sharing, denoted as SemStorm $+($ Off $)$. Compared to SemStorm $+($ Off $)$, SemStorm + showed up to $25 \%$ performance enhancement for queries with low-selectivity common patterns such as $F 2$ and $F 6$.

4.2.4 Varying the Number of rdfs:range Axioms. This task studied the effectiveness of the semantic optimizations that eliminate chain-joins in UCQs. For this study, we disabled the reformulation in the implementation of SemStorm + and measured its impact. The SemStorm + with reformulations and without reformulations were denoted as SemStorm + $(w S)$ and SemStorm + (woS $)$, respectively. We also built custom query sets for this task. A query set $J$ s were constructed from $J 0$, a star pattern query that consists of two triple patterns: the first triple pattern (tp1) includes Property rdf:type and Object ub:Course, which was later expanded into 4 different triple patterns, and (ii) the second triple pattern (tp2) contains Property rdf:type with bounded/unbound Object. We also adjusted LUBM's ontology so that all $J$ queries only have two branches: the branch that contains $J O$ and the branch that includes a reformulation of $J O$ using rdfs:range axioms for ub:Course. The second branch therefore contained a single chain-join. $J 1$ had the reformulated triple pattern the with lowest selectivities while $J 3$ had the one with the highest selectivities. We also compared with Hive( $U)$ to see the performance of these queries using default rewritings. Fig. 14 shows the execution time of these queries. The overall trend was that our semantic optimizations were particularly effective for queries with low selectivities, i.e., the performance gaps between SemStorm+ $(w S)$ and SemStorm $+(w o S)$ was the largest for $J 1$.

\subsection{Additional Experimental Discussion}

We include here some insight into data transfer costs for different approaches. We use the I/O costs (HDFS reads) as a useful metric here, given that the intermediate results are written and read from disk during the shuffle phase. Due to lack of space, we present (Fig 15) only the results of experiments on sharing common expressions (as the results of executing the union branches separately can easily be anticipated). Generally, SemStorm + , Hive(N), and Hive(M) required a smaller amount of HDFS reads because those approaches shared the evaluation of common subexpressions. SemStorm+(On) particularly used minimum amount of disk I/Os for queries with low-selectivity queries. Hive(D) also shared the evaluation but the amount of HDFS read was very large because it could not use vertical partitioning. Overall, it is expected that Semstorm will maintain a significant advantage over other approaches when queries contain lots of star-join structures (the most common use case). However, for chain-join queries this advantage is likely to diminish. But we should note that the other approaches will also diminish in performance because such graph traversal-like queries are not handled well by algebraic query approaches. In fact, the benchmark did not include such queries, making it difficult to conduct meaningful experiments.

\section{CONCLUSION AND FUTURE WORK}

This paper presents several query rewriting and transformation techniques that enable very complex queries with significantly large number of union and join operations to become more parallelizable over shared nothing processing platforms. These complex queries result from translating logical inferencing required for queries over ontological knowledge bases into query rewritings of FOL formulae. The techniques created opportunities for inter-operator parallelism for frameworks that only offer partitioned parallelism. An evaluation of the proposed techniques showed very promising results. 
In contrast, existing platforms that are built on the traditional relational query processing paradigms have inherent scalability limitations. In addition, some of their requirements such as "schema-first" are prohibitive for big variety, semi-structured data, where a-priori knowledge of all possible "schema-structures", as well as, the time required for marshalling data into such structures is impractical. Finally, the execution plans generated based on relational query processing for complex queries such as ontological queries, translates to too many processing cycles that make processing impractical. The takeaway from these observations, and the outcomes of the techniques proposed in this paper, is that there is need to increase emphasis on developing native systems with optimization strategies that specifically target the nuances of ontological data workloads.

\section{ACKNOWLEDGMENTS}

The work presented in this paper is partially funded by NSF grant IIS-1218277 and CNS-1526113.

\section{REFERENCES}

[1] [n. d.]. Adaptive Compression Buffer Size for Wide Tables in ORC. https://issues.apache.org/jira/browse/HIVE-7250.

[2] [n. d.]. Logical Database Limits. https://docs.oracle.com/cd/B28359_ 01/server.111/b28320/limits003.htm\#i288032.

[3] [n. d.]. Project Webpage for R-Type: A Typing Model for RDF. http: //research.csc.ncsu.edu/coul/RAPID+/SemStorm.

[4] [n. d.]. Queries for Evaluation of SemStorm. https://research.csc.ncsu. edu/coul/RAPID+/SemStorm/comp2_queries.html.

[5] [n. d.]. RDF Schema 1.1. https://www.w3.org/TR/rdf-schema.

[6] [n. d.]. RDFHive: A Distributed RDF Store Based on top of Apache Hive. http://tyrex.inria.fr/rdfhive/home.html.

[7] [n. d.]. Resource Description Framework (RDF). https://www.w3.org/RDF/.

[8] [n. d.]. The Linked Open Data Cloud. https://lod-cloud.net/.

[9] [n. d.]. Welcome to Apache Hadoop! http://hadoop.apache.org.

[10] Jans Aasman. 2006. Allegro Graph: RDF Triple Database. Cidade: Oakland Franz Incorporated (2006).

[11] Fran ois Belleau, Marc-Alexandre Nolin, Nicole Tourigny, Philippe Rigault, Jean Morissette, et al. 2008. Bio2RDF: Towards a Mashup to Build Bioinformatics Knowledge Systems. Journal of biomedical informatics 41 (2008).

[12] Saman Biookaghazadeh, Shujia Zhou, and Ming Zhao. 2017. Kaleido: Enabling Efficient Scientific Data Processing on Big-Data Systems. In In Proc. NAS. 1-10.

[13] Damian Bursztyn, Francois Goasdoue, and Ioana Manolescu. 2015. Optimizing Reformulation-based Query Answering in RDF. In Proc. EDBT.

[14] Diego Calvanese, Giuseppe Giacomo, Domenico Lembo, Maurizio Lenzerini, and Riccardo Rosati. 2007. Tractable Reasoning and Efficient Query Answering in Description Logics: The DL-Lite Family. $J$. Autom. Reason. 39, 3 (Oct. 2007), 385-429.

[15] Jae-Young Chang and Sang goo Lee. 1997. An Optimization of Disjunctive Queries: Union-Pushdown. In Proc. COMPSAC. 356-361.

[16] Orri Erling and Ivan Mikhailov. 2009. RDF Support in the Virtuoso DBMS. In Networked Knowledge - Networked Media. Studies in Computational Intelligence, Vol. 221.

[17] Sinziana Maria Filip. 2014. A Scalable Graph Pattern Matching Engine on Top of Apache Giraph. Master's thesis. Vrije Universiteit.

[18] François Goasdoué, Zoi Kaoudi, Ioana Manolescu, Jorge Quiané-Ruiz, and Stamatis Zampetakis. 2015. CliqueSquare: Flat Plans for Massively Parallel RDF Queries. In Proc. ICDE.
[19] Damien Graux, Louis Jachiet, Pierre Geneves, and Nabil Layaida. 2016. SPARQLGX: Efficient Distributed Evaluation of SPARQL with Apache Spark. In Proc. ISWC.

[20] Yuanbo Guo, Zhengxiang Pan, and Jeff Heflin. 2005. LUBM: A Benchmark for OWL Knowledge Base Systems. Semantic Web Journal (2005).

[21] Jiewen Huang, Daniel J. Abadi, and Kun Ren. 2011. Scalable SPARQL Querying of Large RDF Graphs. PVLDB 4 (2011).

[22] M. Husain, J. McGlothlin, M.M. Masud, L. Khan, and Bhavani Thuraisingham. 2011. Heuristics-Based Query Processing for Large RDF Graphs Using Cloud Computing. 23, 9 (2011), 1312-1327.

[23] Eric Jain, Amos Bairoch, Severine Duvaud, Isabelle Phan, Nicole Redaschi, Baris E Suzek, Maria J Martin, Peter McGarvey, and Elisabeth Gasteiger. 2009. Infrastructure for the Life Sciences: Design and Implementation of the UniProt Website. BMC bioinformatics 10 (2009).

[24] Besat Kassaie. 2017. SPARQL over GraphX. CoRR abs/1701.03091 (2017).

[25] HyeongSik Kim, Padmashree Ravindra, and Kemafor Anyanwu. 2012. Scan-Sharing for Optimizing RDF Graph Pattern Matching on MapReduce. In Proc. CLOUD.

[26] H. Kim, P. Ravindra, and K. Anyanwu. 2017. A semantics-aware storage framework for scalable processing of knowledge graphs on Hadoop. In 2017 IEEE International Conference on Big Data (Big Data). 193-202. https://doi.org/10.1109/BigData.2017.8257927

[27] HyeongSik Kim, Padmashree Ravindra, and Kemafor Anyanwu. 2017. A semantics-aware storage framework for scalable processing of knowledge graphs on Hadoop. In 2017 IEEE International Conference on Big Data, BigData 2017, Boston, MA, USA, December 11-14, 2017. 193-202. https://doi.org/10.1109/BigData.2017.8257927

[28] HyeongSik Kim, Padmashree Ravindra, and Kemafor Anyanwu. 2017. Type-based Semantic Optimization for Scalable RDF Graph Pattern Matching. In Proc. WWW. 785-794.

[29] Atanas Kiryakov, Damyan Ognyanov, and Dimitar Manov. 2005. Web Information Systems Engineering - WISE 2005 Workshops. Chapter OWLIM - A Pragmatic Semantic Repository for OWL, 182-192.

[30] Wangchao Le, Anastasios Kementsietsidis, Songyun Duan, and Feifei Li. 2012. Scalable Multi-query Optimization for SPARQL. In Proc. ICDE. 666-677.

[31] Kisung Lee and Ling Liu. 2013. Scaling Queries over Big RDF Graphs with Semantic Hash Partitioning. Proc. VLDB Endow. 6 (2013).

[32] B. McBride. 2002. Jena: A Semantic Web Toolkit. Internet Computing, IEEE 6 (2002).

[33] N. Papailiou, I. Konstantinou, D. Tsoumakos, P. Karras, and N. Koziris. 2013. H2RDF+;: High-performance Distributed Joins Over Large-scale RDF Graphs. In Proc. Big Data.

[34] Minh-Duc Pham, Linnea Passing, Orri Erling, and Peter Boncz. 2015. Deriving an Emergent Relational Schema from RDF Data. In Proc. $W W W$.

[35] Eric Prud'Hommeaux, Andy Seaborne, et al. 2008. SPARQL query language for RDF. W3C recommendation 15 (2008).

[36] Padmashree Ravindra, HyeongSik Kim, and Kemafor Anyanwu. 2011. An Intermediate Algebra for Optimizing RDF Graph Pattern Matching on MapReduce. In Proc. ESWC.

[37] Russ Rew and Glenn Davis. 1990. NetCDF: an interface for scientific data access. IEEE Computer Graphics and Applications 10, 4 (1990), $76-82$.

[38] Alexander Schatzle, Martin Przyjaciel-Zablocki, and Georg Lausen. 2011. PigSPARQL: Mapping SPARQL to Pig Latin. In Proc. SWIM.

[39] Alexander Schatzle, Martin Przyjaciel-Zablocki, Simon Skilevic, and Georg Lausen. 2016. S2RDF: RDF Querying with SPARQL on Spark. Proc. VLDB Endow. 9, 10 (2016), 804-815. 
[40] Heiner Stuckenschmidt and Jeen Broekstra. 2005. Time-Space Tradeoffs in Scaling up RDF Schema Reasoning. In Proc. WISE. 172-181.

[41] Jacopo Urbani, Spyros Kotoulas, Eyal Oren, and Frank Harmelen. 2009. Scalable Distributed Reasoning Using MapReduce. In Proc. ISWC. 634-649.

[42] Jacopo Urbani, Frank van Harmelen, Stefan Schlobach, and Henri Bal. 2011. QueryPIE: Backward Reasoning for OWL Horst over Very Large Knowledge Bases. In Proc. ISWC. 730-745.
[43] Kai Zeng, Jiacheng Yang, Haixun Wang, Bin Shao, and Zhongyuan Wang. 2013. A Distributed Graph Engine for Web Scale RDF Data. In Proc. PVLDB. 265-276.

[44] Xiaofei Zhang, Lei Chen, Yongxin Tong, and Min Wang. 2013. EAGRE: Towards Scalable I/O Efficient SPARQL Query Evaluation on the Cloud. In Proc. ICDE. 0-0.

[45] Lei Zou, Jinghui Mo, Lei Chen, M. Tamer Ozsu, and Dongyan Zhao. 2011. gStore: Answering SPARQL Queries via Subgraph Matching. Proc. VLDB Endow. 4 (2011). 


\section{Appendix: Artifact Description/Artifact Evaluation}

\section{SUMMARY OF THE EXPERIMENTS REPORTED}

Environment: Evaluation was conducted on 45-node Hadoop clusters in University's virtual computing lab, with each node equipped with Xeon dual core x86 CPU (2.33 GHz), 4GB RAM, and 40GB HDD. While cluster sizes may appear larger than necessary, the 45-node cluster made available 900GB (20GB per node) which was required to process and store large intermediate data generated while processing UCQs. All results were averaged over three or more trials.

Dataset: Our testbed consists of Lehigh University Benchmark (LUBM) benchmark datasets, whichare synthesized based on an ontology from academic domain. We employed LUBM 1k, LUBM datasets with $1 \mathrm{k}$ university $(23.8 \mathrm{~GB}, 138 \mathrm{M}$ Triples). Testbed queries were adapted from LUBM benchmark queries to incorporate scenarios on varying characteristics of UCQs such as sharing the evaluation of common expressions and the impact of semantic optimizations. Query selectivities were also varied by binding Object fields of triple patterns (high-selectivity) and evaluating same query with unbound Object (low selectivity).

Approach: We employed a recent stable version of Apache Hive(1.2.2) to simulate the rewriting techniques. Since Hive does not use SPARQL as query language, we translated SPARQL queries into equivalent HiveQL queries. To simulate these approaches, RDF triples were loaded into HDFS and imported into a ternary table T . As optimizations, all approaches except the Hive(D) employed vertical partitioning, which partitions table $\mathrm{T}$ partitioned based on Property types using a map-only job per Property type. The reformulation technique for eliminating schema triple patterns are also applied to all other approaches so that the number of union branches to be evaluated can be equal for all.

\section{ARTIFACT AVAILABILITY}

Software Artifact Availability: There are no author-created software artifacts.

Hardware Artifact Availability: There are no author-created hardware artifacts.

Data Artifact Availability: There are no author-created data artifacts.

Proprietary Artifacts: None of the associated artifacts, authorcreated or otherwise, are proprietary.

List of URLs and/or DOIs where artifacts are available: 